\title{
RIQUEZA DE CHRYSOMELIDAE (COLEOPTERA) EN EL CERRO EL DIENTE, SAN CARLOS, TAMAULIPAS, MÉXICO
}

\section{URIEl JEShuA SÁNCHEZ-REYES ${ }^{1}$, SANTIAgo NIÑO-MALDONADO²*, EdMAR MELÉNDEZ-JARAMILLO ${ }^{1}$, VANNIA dEL CARMEN GÓMEZ-MORENO² \& JoNATHAN EdgaRdo BANDA-HERNÁNDEZ ${ }^{1 \dagger}$}

\author{
${ }^{1}$ Instituto Tecnológico de Ciudad Victoria, Boulevard Emilio Portes Gil No.1301, C.P. 87010. Ciudad Victoria, \\ Tamaulipas, México.<uriel_elf3@hotmail.com> \\ ${ }^{2}$ Universidad Autónoma de Tamaulipas, Facultad de Ingeniería y Ciencias, Centro Universitario Victoria, C.P. \\ 87149. Ciudad Victoria, Tamaulipas, México. <coliopteranino@hotmail.com>
}

Recibido: 07/08/2013; aceptado:20/08/2014

Sánchez-Reyes, U. J., Niño-Maldonado, S., Meléndez-Jaramillo, E., Gómez-Moreno, V. del C. \& Banda-Hernández, J. E. 2015. Riqueza de Chrysomelidae (Coleoptera) en el Cerro El Diente, San Carlos, Tamaulipas, México. Acta Zoológica Mexicana (n. s.), 31(1): 10-22.

RESUMEN. Se realizó un inventario faunístico en el Cerro El Diente, Tamaulipas, para contribuir al conocimiento de la fauna de Chrysomelidae en México. El estudio se llevó a cabo entre marzo y septiembre de 2012; la colecta de los ejemplares se realizó en transectos altitudinales utilizando el método directo. En total se analizaron seis tipos de vegetación, en altitudes de 380 a 1200 msnm. Se obtuvieron 290 individuos, pertenecientes a 74 especies de 50 géneros y seis subfamilias. Este es uno de los primeros estudios de Chrysomelidae realizados en el noreste de México, documentándose ocho nuevos registros para Tamaulipas (Babia costalisdebaja, Diplacaspis prosternalis, Chrysogramma septempunctata, Disonycha teapensis, Margaridisa atriventris, Metrioidea varicornis, Miraces aeneipennis y Acalymma flavovittatum) y uno para México (Malacorhinus acaciae).

Palabras clave: Crisomélidos, Diversidad, Distribución.

\section{INTRODUCCIÓN}

La familia Chrysomelidae es uno de los grupos con mayor riqueza específica dentro del orden Coleoptera, con más de 35,000 especies descritas a nivel mundial, principalmente de hábitos fitófagos (Riley et al. 2002, Jolivet et al. 2009). Muchos crisomélidos son de importancia económica, ya que se convierten en plagas de plantas cultivadas (Ding et al. 2007, Meissle et al. 2009), mientras que otras especies pueden emplearse como controladores biológicos (Syrett et al. 1996); en el ámbito ecológico, los crisomélidos forman un eslabón importante de las redes tróficas (Farrell \& Erwin 1988, Basset \& Samuelson 1996, Eben \& Barbercheck 1996). Además, la gran riqueza específica en esta familia y su rol como grupo funcional fitófago, los convierten en un potencial indicador de la biodiversidad de una región (Farrell \& Erwin 1988, Kalaichelvan \& Verma 2005, Baselga \& Novoa 2007, Aslan \& Ayvaz 2009), la calidad ambiental (Linzmeier et al. 2006), y co-
Sánchez-Reyes, U. J., Niño-Maldonado, S., Meléndez-Jaramillo, E., Gómez-Moreno, V. del C. \& Banda-Hernández, J. E. 2015. Chrysomelidae (Coleoptera) species richness in Cerro El Diente, San Carlos, Tamaulipas, Mexico. Acta Zoológica Mexicana (n. s.), 31(1): 10-22.

ABSTRACT. A faunistic study was conducted at Cerro El Diente, Tamaulipas, to contribute to the knowledge of the fauna of Chrysomelidae in Mexico. The study was conducted between March and September 2012; specimens were collected in altitudinal transects by using direct method. We analyzed six vegetation types at altitudes from 380 to 1200 meters. In total, 290 specimens were collected, belonging to 74 species of 50 genera and six subfamilies. This is one of the first studies conducted at Northeast Mexico, and includes eight new records for Tamaulipas (Babia costalisdebaja, Diplacaspis prosternalis, Chrysogramma septempunctata, Disonycha teapensis, Margaridisa atriventris, Metrioidea varicornis, Miraces aeneipennis and Acalymma flavovittatum), and one for Mexico (Malacorhinus acaciae).

Key words: Leaf beetles, Diversity, Distribution.

mo un taxón para el monitoreo de cambios en áreas naturales (Staines \& Staines 2001, Flowers \& Hanson 2003).

En México, uno de los estudios que reúne gran parte de la información actual sobre riqueza y distribución de Chrysomelidae es el de Ordóñez-Reséndiz et al. (2014), quienes señalan la presencia de 2,174 especies en el país. No obstante, a nivel nacional se desconoce prácticamente cualquier aspecto relacionado con los patrones ecológicos de Chrysomelidae, lo cual obstaculiza la utilización de este grupo como indicador de diversidad, a pesar de la importancia y potencial que presenta (Furth et al. 2003).

Además del trabajo realizado por Ordóñez-Reséndiz et al. (2014), en el país se cuenta solamente con algunos estudios faunísticos enfocados a ciertas subfamilias o tribus, como Chrysomelinae (Burgos-Solorio \& AnayaRosales 2004), Alticini (Furth 2004, 2006, 2009, 2013), Cassidinae (Martínez-Sánchez et al. 2010) o Clytrini (Medvedev et al. 2012); otros autores han analizado a la familia Chrysomelidae en extensiones geográficas más 
amplias (Andrews \& Gilbert 2005, Ordoñez-Reséndiz \& López-Pérez 2009, Ordóñez-Reséndiz et al. 2011), y en áreas naturales protegidas del noreste de México como la Reserva de la Biósfera El Cielo (Niño-Maldonado et al. 2005) o Altas Cumbres (Sánchez-Reyes et al. 2013) en Tamaulipas; sin embargo, es evidente que aún resta gran extensión del territorio mexicano por explorar. En relación a ello, una región trascendente desde el punto de vista biológico y ecológico en México es el Cerro El Diente, que pertenece a la Sierra de San Carlos al noroeste del estado de Tamaulipas (Figura 1). El área forma parte de un macizo montañoso aislado en la Llanura Costera del
Golfo y por ello se le considera como una isla biogeográfica o isla ecológica; además representa el límite boreal del bosque mesófilo en México (Briones-Villarreal 1991, Arriaga et al. 2000), e incluye puntos con prioridad alta a extrema para la conservación de acuerdo a CONABIO et al. (2007) en donde no se han desarrollado estudios faunísticos y/o ecológicos para ningún grupo entomológico. Con base en lo anterior, los objetivos de este estudio fueron 1) realizar un inventario preliminar de las especies de Chrysomelidae en el Cerro El Diente, San Carlos, Tamaulipas, y 2) aportar información básica sobre la distribución espacial y temporal de las especies en el área.
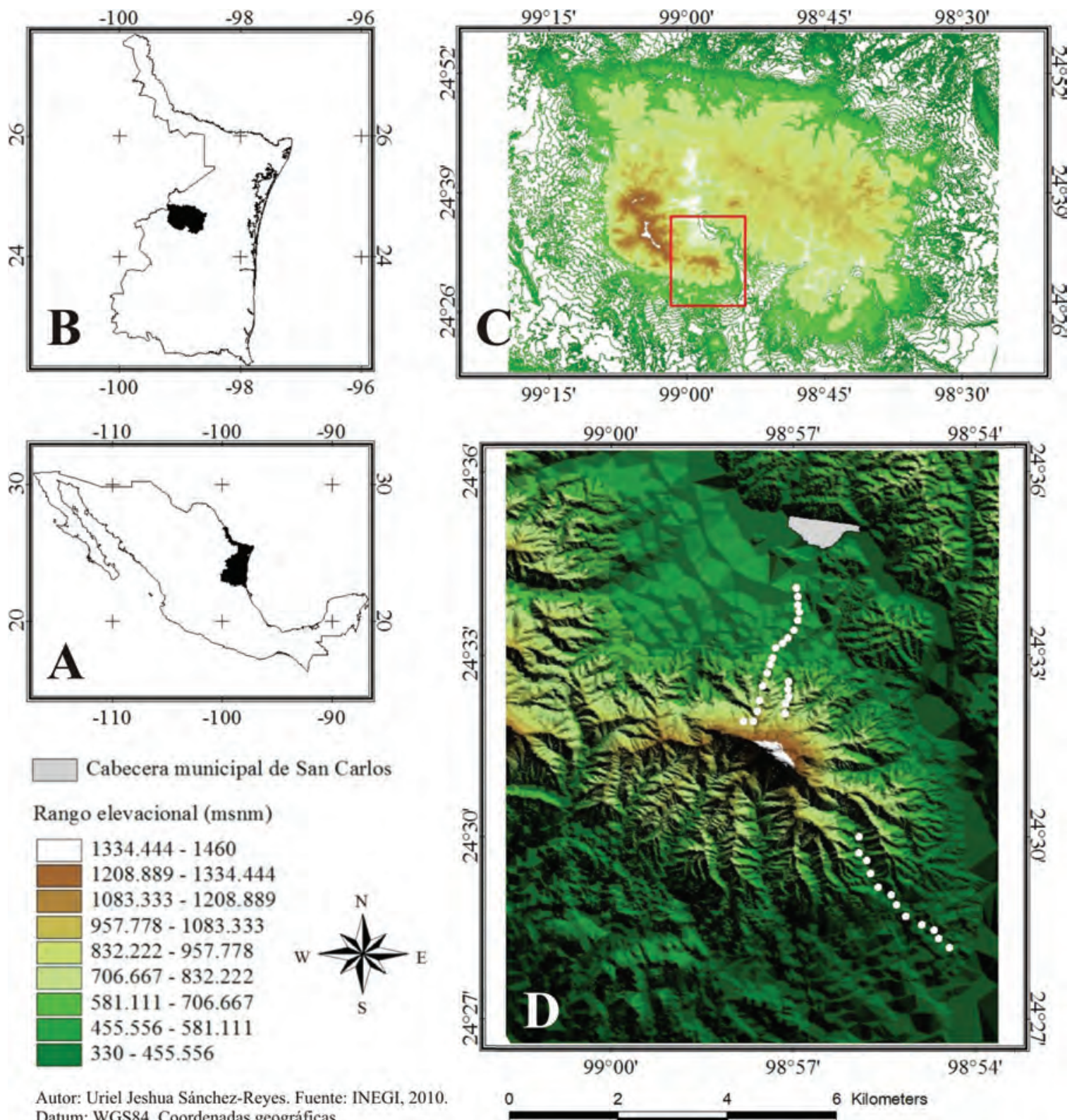

Figura 1. Ubicación del Cerro El Diente, en la Sierra de San Carlos, Tamaulipas, México. A) México; B) Sierra de San Carlos en Tamaulipas; C) Localización del Cerro El Diente dentro de la Sierra de San Carlos; D) Detalle del Cerro El Diente y ubicación aproximada de los transectos de colecta (líneas punteadas en color blanco). 


\section{MATERIAL Y MÉTODOS}

Área de estudio. El Cerro El Diente forma parte de la Sierra de San Carlos, al noreste de México en el estado de Tamaulipas (Fig. 1). El clima es semicálido a templado, con periodo seco en verano y una temperatura media anual superior a los $18{ }^{\circ} \mathrm{C}$, presentando el mes más frío entre -3 y $18^{\circ} \mathrm{C}$. La precipitación media anual es de 1000 mm, y puede alcanzar los 1200 en las partes más húmedas, mientras que la precipitación en el mes más seco es menor a $40 \mathrm{~mm}$. Los vientos dominantes provienen del sureste, originando lluvias durante todo el año a excepción de invierno, donde dominan vientos del norte (Arriaga et al. 2000, Cavazos-Camacho 2000).

Las comunidades vegetales se encuentran en buen estado de conservación y comprenden principalmente bosques de encino y encino-pino, matorral submontano, matorral espinoso tamaulipeco, así como algunas áreas de selva baja y vegetación riparia (Martínez 1998, Briones-Villarreal 1991). En las partes más altas del Cerro El Diente, en exposición norte del macizo montañoso Bufa El Diente, se encuentra el bosque deciduo templado de montaña (bosque mesófilo de montaña) (Briones-Villarreal 1991), lo cual representa el límite boreal de este tipo de vegetación en México (Arriaga et al. 2000).

Colecta y procesamiento de ejemplares. Para la colecta de los ejemplares se utilizó el método directo (mediante aspirador o en forma manual), además de red entomológica aérea, colocando los ejemplares en alcohol etílico al $70 \%$. El muestreo se realizó a lo largo de tres transectos de entre cinco a siete kilómetros, en un horario de 9:00 a 16:00 hrs, comprendiendo un gradiente altitudinal de 380 hasta 1200 msnm (Fig. 1D); en total se llevaron a cabo 19 colectas entre marzo a septiembre de 2012. La determinación taxonómica se efectuó mediante literatura especializada y por comparación con los ejemplares de la colección de la Facultad de Ingeniería y Ciencias de la Universidad Autónoma de Tamaulipas. El arreglo taxonómico empleado corresponde a la clasificación propuesta por Riley et al. (2013); todos los ejemplares se depositaron en la colección de la Facultad de Ingeniería y Ciencias de la Universidad Autónoma de Tamaulipas.

Análisis de datos. Para determinar el número potencial de especies en la zona de estudio se emplearon los estimadores no paramétricos de Chao 2 y Jackknife (1 y 2), ya que se tomó en cuenta solamente la presencia-ausencia de las especies y además son estimadores robustos en el cálculo de la estimación mínima de la riqueza, por lo cual se recomienda su utilización como medidas recurrentes en análisis de biodiversidad (Magurran 2004, Hortal et al. 2006, Gotelli \& Colwell 2011); los estimadores se calcularon con 100 aleatorizaciones (sin reemplazamien- to) mediante el programa EstimateS 8.2 (Colwell 2009), tomando como base el número de especies encontradas por unidad de muestreo (fecha de colecta). Además se contabilizó el número de especies raras, siendo aquellas para las que se obtuvo un solo ejemplar (singletons) y dos ejemplares (doubletons) (Magurran 2004).

Para la medición de la diversidad alfa se utilizaron el índice de diversidad de Simpson (1/D) y el índice de diversidad de Shannon $\left(\mathrm{H}^{\prime}\right)$, así como su valor transformado $\left(\mathrm{e}^{\mathrm{H}^{\prime}}\right)$ (Magurran 2004, Jost 2006), empleando el programa PAST versión 1.94b (Hammer et al. 2001).

En el análisis espacial y temporal se consideró la presencia-ausencia de las especies en cada estrato altitudinal, tipo de vegetación, y mes de colecta; también se cuantificó el número de especies exclusivas a cada variable, es decir, aquellas que se registraron únicamente en una comunidad vegetal, altitud o mes. Para determinar la semejanza en la composición faunística entre tipos de vegetación y meses de colecta, se empleó el índice de Sorensen (Dice), ya que se tomó en cuenta solamente la incidencia (presencia/ausencia) de las especies (Magurran 2004).

\section{RESULTADOS}

Se capturaron un total de 290 ejemplares de Chrysomelidae, distribuidos en 50 géneros y 74 especies, de las cuales $30(40.54 \%)$ fueron singletons y 14 (18.9\%) fueron doubletons (ver Sg y Db, Cuadro 1). De la riqueza total observada, 32 especies (43.24\%) corresponden a la subfamilia Galerucinae, 20 (27.02\%) a Cryptocephalinae, ocho (10.81\%) a Cassidinae, siete (9.45\%) a Eumolpinae, cuatro (5.4\%) a Chrysomelinae y solo tres especies (4.05\%) a Criocerinae (Fig. 2). La riqueza estimada fue de 117 a 143 especies potenciales en el área de estudio (Fig. 3); por lo tanto, la riqueza total observada representa entre el 51.61 al 63.44\% del estimado (Cuadro 2). La diversidad calculada fue 3.78 para el índice de Shannon $\left(\mathrm{H}^{\prime}\right)$, 43.81 de acuerdo al valor transformado de Shannon $\left(\mathrm{e}^{\mathrm{H}^{\mathrm{f}}}\right)$, y 28.78 para el índice de diversidad de Simpson (1/D).

Altitudinalmente, la mayor riqueza específica (18 especies) se registró a los 550 metros, así como a los 800 (15 especies) y 780 metros (14 especies) (Fig. 4); por otra parte, tres estratos altitudinales presentaron una sola especie: Exema conspersa se observó a los 400 msnm, Smaragdina agilis a $900 \mathrm{msnm}$ y Anomoea rufifrons mutabilis a $950 \mathrm{msnm}$ (Cuadro 1). De la riqueza total observada en el Cerro el Diente, 50 especies fueron colectadas solo en un estrato altitudinal; de éstas, la mayor cantidad (13 especies) se registró exclusivamente a los $550 \mathrm{msnm}$ (Fig. 4); en cambio, Cryptocephalus trizonatus y Diplacaspis prosternalis se presentaron únicamente a 540 msnm (Cuadro 1). 
Cuadro 1. Listado taxonómico preliminar de Chrysomelidae en el Cerro El Diente, San Carlos, Tamaulipas, México. El arreglo de las especies se presenta en base a Riley et al. (2003).

\begin{tabular}{|c|c|c|c|}
\hline & Vegetación & Altitud & Mes de colecta \\
\hline \multicolumn{4}{|l|}{ CASSIDINAE Gyllenhal, 1813} \\
\hline \multicolumn{4}{|l|}{ Tribu Chalepini Weise, 1910} \\
\hline Brachycoryna pumila Guérin, $1844^{\mathrm{Db}}$ & $\mathrm{BE}$ & 780 & Mar, Sep \\
\hline Sumitrosis inaequalis (Weber, 1801) & $\mathrm{BE}$ & 780 & Jun, Jul, Sep \\
\hline \multicolumn{4}{|l|}{ Tribu Cassidini Gyllenhal, 1813} \\
\hline Charidotella sexpunctata (Fabricius, 1781) ${ }^{\mathrm{Db}}$ & $\mathrm{RP}$ & 440 & Ago \\
\hline Gratiana lutescens (Boheman, 1854) $^{\mathrm{sg}}$ & MS, BE & 600 & Ago \\
\hline Metrionella bilimeki Spaeth, 1932 Db & $\mathrm{BP}$ & 1100 & Sep \\
\hline Helocassis clavata (Fabricius, 1798) ${ }^{\mathrm{Sg}}$ & MS, BE & $600-750$ & Ago \\
\hline Helocassis crucipennis (Boheman, 1855) ${ }^{\mathrm{sg}}$ & ME & 550 & Ago \\
\hline \multicolumn{4}{|l|}{ Tribu Mesomphaliini Hope, 1840} \\
\hline Ogdoecosta juvenca (Boheman, 1854) & BP & 1,100 & Ago, Sep \\
\hline \multicolumn{4}{|l|}{ CHRYSOMELINAE Latreille, 1802} \\
\hline \multicolumn{4}{|l|}{ Tribu Chrysomelini Latreille, 1802} \\
\hline \multicolumn{4}{|l|}{ Subtribu Doryphorina Motschulsky, 1860} \\
\hline Labidomera suturella Chevrolat, 1844 Db & BE, BP & $780-1100$ & Mar \\
\hline \multicolumn{4}{|l|}{ Subtribu Chrysomelina Latreille, 1802} \\
\hline Chrysomela texana (Schaeffer, 1919) ${ }^{\mathrm{sg}}$ & $\mathrm{RP}$ & 440 & Ago \\
\hline Plagiodera semivittata Stål, 1860 & ME, MS, BE & $550-800$ & Mar, Jul, Ago, Sep \\
\hline Plagiodera thymaloides Stål, 1860 sg & $\mathrm{BE}$ & 780 & Mar \\
\hline \multicolumn{4}{|l|}{ CRIOCERINAE Latreille, 1807} \\
\hline \multicolumn{4}{|l|}{ Tribu Lemini Heinze, 1962} \\
\hline Lema opulenta Gemminger \& Harold, 1874 Sg & MS, BE & $750-800$ & Ago \\
\hline Neolema quadriguttata White, 1993 sg & SB & 630 & Jun, Jul \\
\hline Neolema sp.1 & MS, BE & $750-800$ & Sep \\
\hline \multicolumn{4}{|l|}{ CRYPTOCEPHALINAE Gyllenhal, 1813} \\
\hline \multicolumn{4}{|l|}{ Tribu Cryptocephalini Gyllenhal, 1813} \\
\hline \multicolumn{4}{|l|}{ Subtribu Pachybrachina Chapuis, 1874} \\
\hline Pachybrachis sp.1 & $\mathrm{RP}$ & 440 & Ago, Sep \\
\hline Pachybrachis sp. $2^{\text {sg }}$ & $\mathrm{RP}$ & 440 & Ago \\
\hline Pachybrachis sp.3 & RP, ME, MS, BE & $440-780$ & Jul, Ago, Sep \\
\hline Pachybrachis sp.4 & $\mathrm{RP}$ & 440 & Ago \\
\hline \multicolumn{4}{|l|}{ Subtribu Monachulina Leng, 1920} \\
\hline Lexiphanes guerini (Perbosc, 1839) & RP & 440 & Ago, Sep \\
\hline \multicolumn{4}{|l|}{ Subtribu Cryptocephalina Gyllenhal, 1813} \\
\hline Cryptocephalus guttulatus Olivier, 1808 & BP & 1,100 & Ago, Sep \\
\hline Cryptocephalus trizonatus Suffrian, 1858 sg & $\mathrm{ME}$ & 540 & Abr, May \\
\hline Cryptocephalus umbonatus Schaeffer, 1906 Sg & MS, BE & 680 & Ago \\
\hline Diachus auratus (Fabricius, 1801) & RP, MS, BE & $440-780$ & Ago, Sep \\
\hline \multicolumn{4}{|l|}{ Tribu Clytrini Lacordaire, 1848} \\
\hline \multicolumn{4}{|l|}{ Subtribu Ischiopachyna } \\
\hline Ischiopachys bicolor proteus Lacordaire, 1848 & ME & 550 & Sep \\
\hline \multicolumn{4}{|l|}{ Subtribu Clytrina Lacordaire, 1848} \\
\hline Anomoea rufifrons mutabilis (Lacordaire, 1848) & ME, MS, BE & $550-950$ & Ago, Sep \\
\hline Smaragdina agilis (Lacordaire, 1848) ${ }^{\mathrm{sg}}$ & MS, BE & 900 & Ago \\
\hline
\end{tabular}


Cuadro 1. Continúa.

\begin{tabular}{|c|c|c|c|}
\hline & Vegetación & Altitud & Mes de colecta \\
\hline \multicolumn{4}{|l|}{ Subtribu Megalostomina Chapuis, 1874} \\
\hline Coscinoptera aeneipennis (LeConte, 1858) & ME, BE & $550-780$ & Ago, Sep \\
\hline Coscinoptera scapularis scapularis (Lacordaire, 1848) ${ }^{\mathrm{sg}}$ & ME & 550 & Ago \\
\hline Coscinoptera tamaulipasi L. Medvedev, 2012 & ME & 550 & Ago, Sep \\
\hline Megalostomis tomentosa apicata Achard, 1926 & $\mathrm{BE}$ & 780 & Sep \\
\hline \multicolumn{4}{|l|}{ Subtribu Babiina Chapuis, 1874} \\
\hline Babia costalisdebaja Moldenke, 1970* Sg & $\mathrm{ME}$ & 550 & Abr, May \\
\hline Babia tetraspilota texana Schaeffer, 1933 sg & ME & 550 & Ago \\
\hline \multicolumn{4}{|l|}{ Tribu Chlamisini Gressitt, 1945} \\
\hline Diplacaspis prosternalis (Schaeffer, 1906)*Db & ME & 540 & Abr, May \\
\hline Exema conspersa (Mannerheim, 1843) & $\mathrm{RP}, \mathrm{ME}$ & $400-440$ & Abr, Ago \\
\hline \multicolumn{4}{|l|}{ EUMOLPINAE Hope, 1840} \\
\hline \multicolumn{4}{|l|}{ Tribu Eumolpini Hope, 1840} \\
\hline \multicolumn{4}{|l|}{ Grupo Iphimeites Chapuis, 1874} \\
\hline Brachypnoea sp.1 ${ }^{\mathrm{Db}}$ & MS, BE & $700-800$ & Mar, Ago \\
\hline Brachypnoea sp.2 ${ }^{\mathrm{Db}}$ & ME & 550 & Mar, Sep \\
\hline Brachypnoea sp.3 sg & MS, BE & $700-800$ & Ago \\
\hline Colaspis melancholica Jacoby, $1881^{\mathrm{Db}}$ & $\mathrm{BE}$ & 780 & Sep \\
\hline Zenocolaspis sp.1 & RP & 380 & Abr, May \\
\hline \multicolumn{4}{|l|}{ Tribu Adoxini Baly, 1863} \\
\hline \multicolumn{4}{|l|}{ Grupo Leprotites Chapuis, 1874} \\
\hline Xanthonia sp.1 & MS, BE & $700-800$ & Mar, Sep \\
\hline Xanthonia sp.2 & MS, BE & $700-800$ & Mar, Sep \\
\hline \multicolumn{4}{|l|}{ GALERUCINAE Latreille, 1802} \\
\hline \multicolumn{4}{|l|}{ Tribu Alticini Newman, 1835} \\
\hline Acallepitrix sp. $1^{\mathrm{sg}}$ & ME & 550 & Ago \\
\hline Altica sp. $1^{\mathrm{Db}}$ & $\mathrm{RP}$ & 380 & Abr, May \\
\hline Asphaera abdominalis (Chevrolat, 1834) ${ }^{\mathrm{sg}}$ & ME & 550 & Ago \\
\hline Asphaera sp. $1^{\mathrm{Db}}$ & $\mathrm{RP}$ & 440 & Ago \\
\hline Centralaphthona diversa (Baly, 1877) & $\mathrm{SB}, \mathrm{ME}, \mathrm{MS}, \mathrm{BE}$ & $550-800$ & Jun, Jul, Ago \\
\hline Chaetocnema sp.1 & SB & 630 & Jun, Jul \\
\hline Chaetocnema sp.2 & SB, MS, BE & $630-800$ & Jul, Sep \\
\hline Chaetocnema sp.3 $3^{\mathrm{Db}}$ & MS, BE & $700-800$ & Sep \\
\hline Chaetocnema sp.4 sg & $\mathrm{BE}$ & 780 & Jun, Jul \\
\hline Chrysogramma octomaculata Jacoby, 1885 & MS, BE & $700-800$ & Mar \\
\hline Chrysogramma septempunctata Jacoby, 1891* sg & MS, BE & 750 & Ago \\
\hline Dibolia sp. $1^{\text {sg }}$ & SB & 630 & Jul \\
\hline Disonycha antennata Jacoby, 1884 sg & ME & 550 & Ago \\
\hline Disonycha glabrata (Fabricius, 1781) ${ }^{\mathrm{sg}}$ & MS, BE & & Ago \\
\hline Disonycha stenosticha Schaeffer, 1931 & MS, BE & $700-800$ & Mar, Sep \\
\hline Disonycha teapensis Blake, 1933* sg & MS, BE & & Ago \\
\hline Longitarsus sp. $1^{\mathrm{sg}}$ & $\mathrm{ME}$ & 550 & Ago \\
\hline Longitarsus sp. $2^{\mathrm{sg}}$ & MS, BE & $700-800$ & Sep \\
\hline Lupraea sp. $1^{\mathrm{Db}}$ & MS, BE & & Ago \\
\hline Macrohaltica jamaicensis (Fabricius, 1792) ${ }^{\mathrm{sg}}$ & $\mathrm{RP}$ & 380 & Abr, May \\
\hline
\end{tabular}


Cuadro 1. Continúa.

\begin{tabular}{|c|c|c|c|}
\hline & Vegetación & Altitud & Mes de colecta \\
\hline \multicolumn{4}{|l|}{ GALERUCINAE Latreille, 1802} \\
\hline \multicolumn{4}{|l|}{ Tribu Alticini Newman, 1835} \\
\hline Margaridisa atriventris (F. E. Melsheimer, 1847)* & MS, BE & $700-800$ & Jun, Jul, Sep \\
\hline Margaridisa sp.2 & MS, BE & $700-800$ & Sep \\
\hline Syphrea sp. $1^{\text {sg }}$ & $\mathrm{BE}$ & 780 & Mar \\
\hline Syphrea sp.2 & $\mathrm{BE}$ & 780 & Mar, Jul, Ago, Sep \\
\hline Systena sp.1 & $\mathrm{RP}$ & 380 & Abr, May \\
\hline \multicolumn{4}{|l|}{ Tribu Galerucini Latreille, 1802} \\
\hline \multicolumn{4}{|l|}{ Grupo Coelomerites Chapuis, 1875} \\
\hline Miraces aeneipennis Jacoby, 1888* & ME & 550 & Ago \\
\hline \multicolumn{4}{|l|}{ Tribu Metacyclini Chapuis, 1875} \\
\hline Malacorhinus acaciae (Schaeffer, 1906)** sg & $\mathrm{RP}$ & 380 & Abr, May \\
\hline \multicolumn{4}{|l|}{ Tribu Luperini Chapuis, 1875} \\
\hline \multicolumn{4}{|l|}{ Subtribu Diabroticina Chapuis, 1875} \\
\hline \multicolumn{4}{|l|}{ Grupo Diabroticites Chapuis, 1785} \\
\hline Acalymma flavovittatum (Baly, 1886)* Sg & ME & 550 & Ago \\
\hline Diabrotica balteata LeConte, $1865^{\mathrm{Db}}$ & $\mathrm{RP}$ & 440 & Ago, Sep \\
\hline \multicolumn{4}{|l|}{ Grupo Cerotomites Chapuis, 1875} \\
\hline Cyclotrypema furcata (Olivier, 1808) & RP, MS, BE & $380-750$ & Abr, Ago \\
\hline \multicolumn{4}{|l|}{ Subtribu Luperina Chapuis, 1875} \\
\hline \multicolumn{4}{|l|}{ Grupo Scelidites Chapuis, 1875} \\
\hline Scelida metallica Jacoby, $1888^{\mathrm{Db}}$ & MS, BE & 680,750 & Sep \\
\hline \multicolumn{4}{|l|}{ Grupo Monoleptites Chapuis, 1875} \\
\hline Metrioidea varicornis (J. L. LeConte, 1868)* sg & $\mathrm{BE}$ & 780 & Mar \\
\hline
\end{tabular}

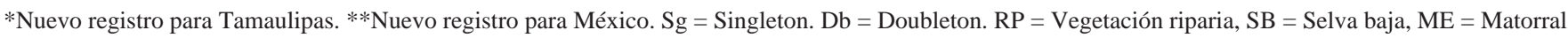
espinoso; $\mathrm{MS}=$ Matorral submontano; $\mathrm{BE}$ = Bosque de encino; $\mathrm{BP}$ = Bosque de pino.

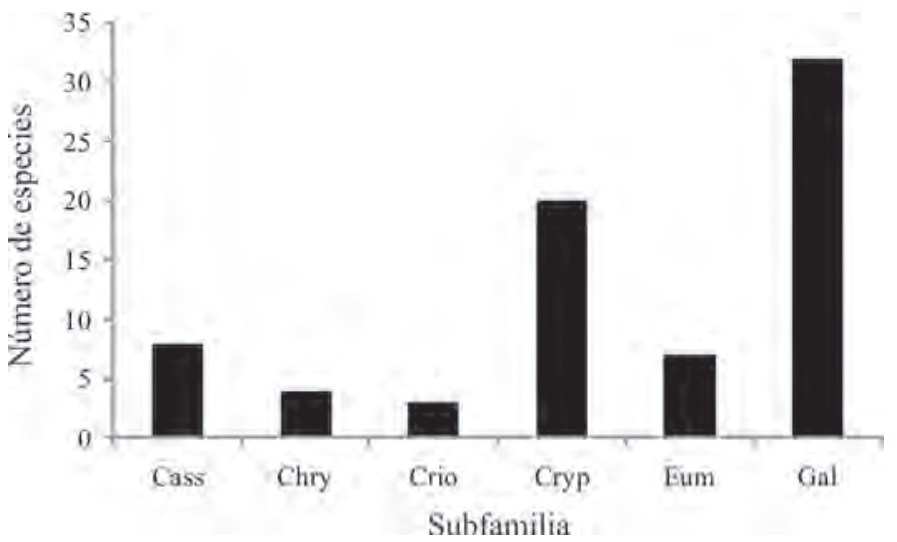

Figura 2. Número de especies por subfamilia de Chrysomelidae en el Cerro El Diente. Cass = Cassidinae, Chry = Chrysomelinae, Crio $=$ Criocerinae, Cryp $=$ Cryptocephalinae, Eum $=$ Eumolpinae, $\mathrm{Gal}=$ Galerucinae.

En relación al tipo de vegetación, el mayor número de especies (39) fue registrado en el bosque de encino, seguido del matorral submontano (28 especies), matorral
Cuadro 2. Valores de riqueza estimada de Chrysomelidae en el Cerro El Diente.

\begin{tabular}{lcc}
\hline Modelo & Riqueza estimada & \% representatividad \\
\hline Chao2 & 137.28 & 53.9 \\
Jackknife1 & 116.63 & 63.44 \\
Jackknife 2 & 143.37 & 51.61 \\
\hline
\end{tabular}

\% obtenido con base en la riqueza observada.

espinoso (21), vegetación riparia (17), selva baja (5) y bosque de pino (4). En total se obtuvieron 43 especies únicas a un tipo de vegetación: los valores más elevados se presentaron en el matorral espinoso tamaulipeco (15 especies) y en la vegetación riparia (13) (Fig. 5). Además, 24 especies fueron registradas en dos comunidades vegetales diferentes, cinco especies se asociaron a tres tipos de vegetación, y solo Pachybrachis sp.3 y Centralaphthona diversa se distribuyeron en cuatro comunidades de plantas (Cuadro 1).

El análisis de similitud indicó un 83\% de semejanza en la composición faunística de Chrysomelidae entre el 


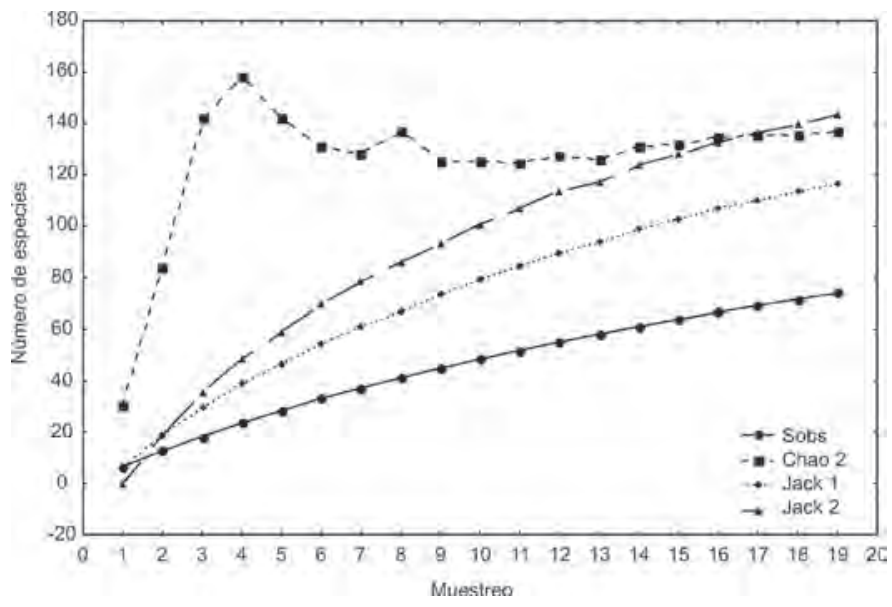

Figura 3. Riqueza potencial de Chrysomelidae en el Cerro El Diente. Sobs = Riqueza observada; Chao2, Jack1, Jack2 = estimadores de riqueza.

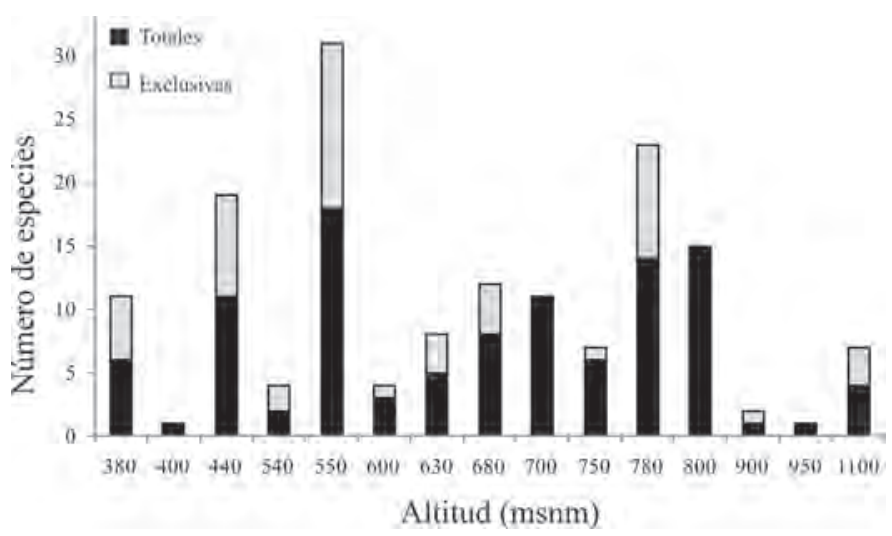

Figura 4. Riqueza específica de Chrysomelidae por punto altitudinal en el Cerro El Diente.

bosque de encino y el matorral submontano, con 28 especies comunes a ambas comunidades vegetales. El resto de las comparaciones entre diferentes tipos de vegetación mostró valores por debajo del $20 \%$ de similitud. No se encontró ninguna especie en común ( $0 \%$ de similitud) entre la selva baja con respecto a la vegetación riparia, ni tampoco entre el bosque de pino con relación a la vegetación riparia, selva baja, matorral espinoso y matorral submontano (Cuadro 3).

De acuerdo al análisis temporal, la mayor riqueza se observó en agosto (40 especies), y con valores menores se presentaron los meses de septiembre (29 especies), marzo (13), julio (11), abril (10), mayo (8) y junio (6). Por otra parte, 39 especies fueron exclusivas a un mes en particular: el valor más elevado se registró en agosto (24 especies exclusivas) y posteriormente septiembre (9), marzo (5) y julio (1) (Fig. 6).

Todas las especies registradas en abril, mayo y junio fueron comunes a otros meses, y la mayor similitud faunís-

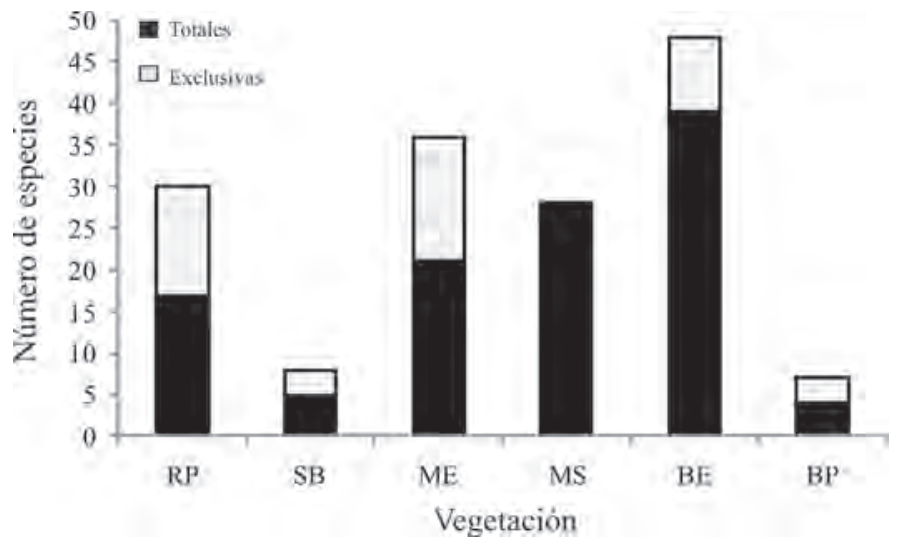

Figura 5. Riqueza específica de Chrysomelidae por tipo de vegetación en el Cerro El Diente. $\mathrm{RP}=$ vegetación riparia, $\mathrm{SB}=$ selva baja, $\mathrm{ME}=$ matorral espinoso, $\mathrm{MS}=$ matorral submontano, $\mathrm{BE}=$ bosque de encino, $\mathrm{BP}=$ bosque de pino.

Cuadro 3. Similitud faunística de Chrysomelidae entre tipos de vegetación en el Cerro El Diente. Valores por encima de la diagonal representan similitud; valores por debajo de la diagonal representan el número de especies compartidas.

\begin{tabular}{lcccccc}
\hline & RP & SB & ME & MS & BE & BP \\
\hline RP & - & 0 & 0.105 & 0.133 & 0.107 & 0 \\
SB & 0 & - & 0.076 & 0.121 & 0.090 & 0 \\
ME & 2 & 1 & - & 0.163 & 0.166 & 0 \\
MS & 3 & 2 & 4 & - & 0.835 & 0 \\
BE & 3 & 2 & 5 & 28 & - & 0.046 \\
BP & 0 & 0 & 0 & 0 & 1 & - \\
\hline
\end{tabular}

RP = Vegetación riparia, SB = Selva baja, ME = Matorral espinoso; MS = Matorral submontano; $\mathrm{BE}=$ Bosque de encino; $\mathrm{BP}=$ Bosque de pino.

tica (88\%) se presentó entre abril y mayo, seguidos de junio y julio (70\%); se obtuvieron valores inferiores para el resto de comparaciones entre los meses (Cuadro 4).

Nuevos registros. En el presente estudio se reporta la ampliación en el rango de distribución de ocho especies hacia el noreste de México, las cuales son registradas por vez primera para Tamaulipas; además, se incluye un nuevo registro para México.

Subfamilia Cryptocephalinae Gyllenhal, 1813

Tribu Clytrini Lacordaire, 1848

Subtribu Babiina Chapuis, 1874

Babia costalisdebaja Moldenke, 1970. Distribución en México: Baja California (Moldenke 1970; Andrews \& Gilbert 2005). Distribución en el Cerro El Diente: abril y mayo, $550 \mathrm{msnm}$, matorral espinoso $\left(24^{\circ} 30^{\prime} 2.64^{\prime \prime} \mathrm{N}\right.$, 9855'58.43”O) (Fig. 7A-3). Observaciones: nuevo registro para Tamaulipas.

Tribu Chlamisini Gressitt, 1946

Diplacaspis prosternalis (Schaeffer, 1906). Distribución en México: sin localidad específica (Wilcox 1975); Baja 
Cuadro 4. Similitud faunística de Chrysomelidae entre meses de colecta en el Cerro El Diente. Valores por encima de la diagonal representan similitud; valores por debajo de la diagonal representan el número de especies compartidas.

\begin{tabular}{lccccccc}
\hline & Marzo & Abril & Mayo & Junio & Julio & Agosto & Septiembre \\
\hline Marzo & - & 0 & 0 & 0 & 0.16 & 0.11 & 0.33 \\
Abril & 0 & - & 0.88 & 0 & 0 & 0.08 & 0 \\
Mayo & 0 & 8 & - & 0 & 0 & 0 & 0 \\
Junio & 0 & 0 & 0 & - & 0.70 & 0.04 & 0.11 \\
Julio & 2 & 0 & 0 & 6 & - & 0.15 & 0.3 \\
Agosto & 3 & 2 & 0 & 1 & 4 & - & 0.34 \\
Septiembre & 7 & 0 & 0 & 2 & 6 & 12 & - \\
\hline
\end{tabular}

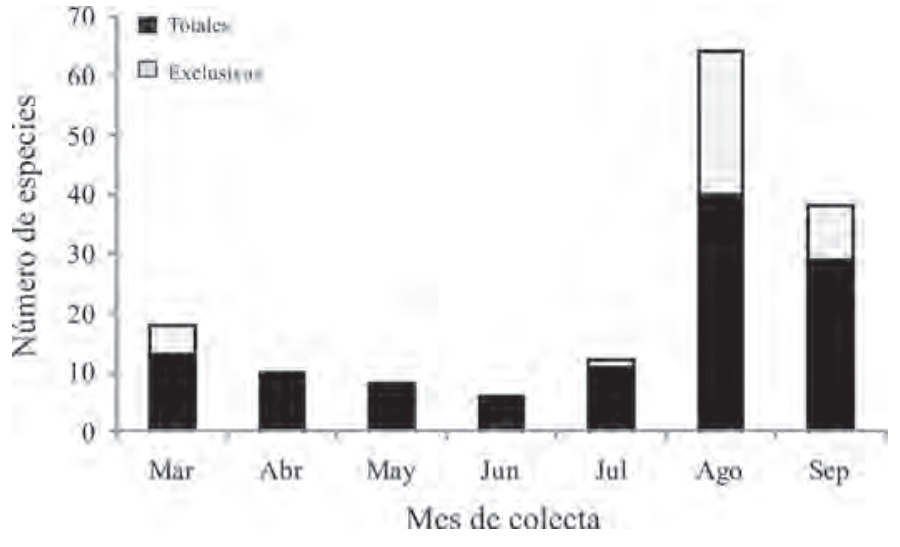

Figura 6. Riqueza específica de Chrysomelidae por mes en el Cerro El Diente.

California (Andrews \& Gilbert 2005). Distribución en el Cerro El Diente: abril y mayo, 540 msnm, matorral espinoso (24²9’53.07’N, 9855’56.82”O) (Fig. 7A-4). Observaciones: nuevo registro para Tamaulipas.

Subfamilia Galerucinae Latreille, 1802

Tribu Alticini Newman, 1835

Chrysogramma septempunctata Jacoby, 1891. Distribución en México: sin registro de localidad (Furth \& Savini 1996), Durango, Morelos, Oaxaca y Puebla (Furth 2006). Distribución en el Cerro El Diente: agosto, 750 msnm, matorral submontano y bosque de encino (Fig. 7B-7) (2432’32.13”N, 9857’26.91”O). Observaciones: nuevo registro para Tamaulipas.

Disonycha teapensis Blake, 1933. Distribución en México: sin registro de localidad (Furth \& Savini 1996, Furth 2004); San Luis Potosí (Blake 1955). Distribución en el Cerro El Diente: agosto, $680 \mathrm{msnm}$, matorral submontano y bosque de encino (24³2'38.89’ N, 9857’28.83”O) (Fig. 7B-6). Observaciones: nuevo registro para Tamaulipas.

Margaridisa atriventris Bechyne, 1958. Distribución en México: Chiapas, Veracruz (Furth 2004, 2006). Distribución en el Cerro El Diente: junio, julio y septiembre, 700 y 800 msnm, matorral submontano y bosque de encino (24³2'6.42”N, 9857'15.72”O; 2432'35.64”N, 98 57’24.80”O) (Fig. 7A-2). Observaciones: nuevo registro para Tamaulipas.

Tribu Galerucini Latreille, 1802

Miraces aeneipennis Jacoby, 1888. Distribución en México: sin registro de localidad (Wilcox 1975). Distribución en el Cerro El Diente: agosto, $550 \mathrm{msnm}$, matorral espinoso (24³3’1.49”'N, 9857’5.84”O) (Fig. 7A-1). Observaciones: nuevo registro para Tamaulipas.

Tribu Metacyclini Chapuis, 1875

Malacorhinus acaciae (Schaeffer, 1906). Distribución en México: sin distribución previa conocida. Se reporta en Texas (Estados Unidos) y América Central (Wilcox 1951, Riley et al. 2003). Distribución en el Cerro El Diente: abril y mayo, $380 \mathrm{msnm}$, vegetación riparia (242'14.01”N, 9854'33.15”O) (Fig. 7B-9). Observaciones: primer registro de la especie en México.

Tribu Luperini Chapuis, 1875

Subtribu Diabroticina Chapuis, 1875

Acalymma flavovittatum (Baly, 1886). Distribución en México: Campeche, Quintana Roo y Yucatán (Munroe \& Smith 1980). Distribución en el Cerro El Diente: agosto, 550 msnm, matorral espinoso (243’ $1.50^{\prime \prime} \mathrm{N}$, 9857’5.84’O) (Fig. 7B-5). Observaciones: nuevo registro para Tamaulipas.

Subtribu Luperina Chapuis, 1875

Metrioidea varicornis Fairmaire 1881. Distribución en México: sin registro de localidad (Wilcox 1975); Baja California (Andrews \& Gilbert 2005). Distribución en el Cerro El Diente: marzo, $780 \mathrm{msnm}$, bosque de encino (24³2'8.40”N, 9857’14.97’'O) (Fig. 7B-8). Observaciones: Nuevo registro para Tamaulipas.

\section{DISCUSIÓN}

En el estado de Tamaulipas, Ordóñez-Reséndiz et al. (2014) señalan la presencia de 93 especies. Tomando en cuenta esa cifra, las 74 especies encontradas indican que el Cerro El Diente alberga el $79.57 \%$ de la riqueza total del estado. Aunado a ello, los estimadores de riqueza su- 


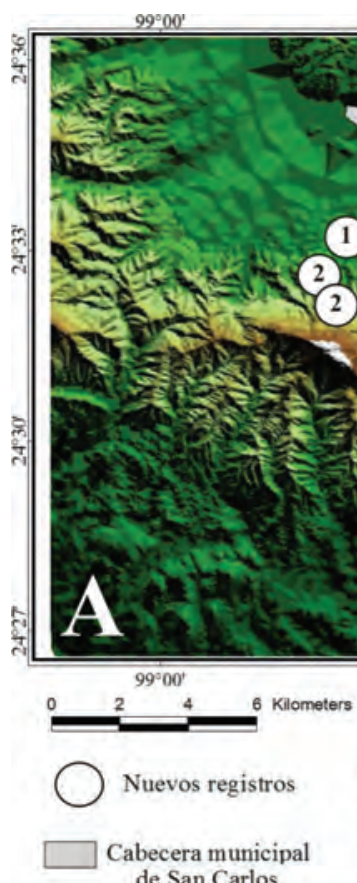

$98^{\circ} 5_{0}$

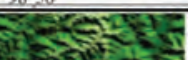

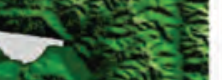

de San Carlos

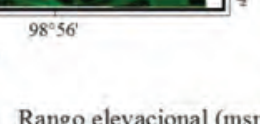

Rango elevacional (msnm) $1334.444-1460$ $1208.889-1334.444$ $1083.333-1208.889$ $957.778-1083.333$

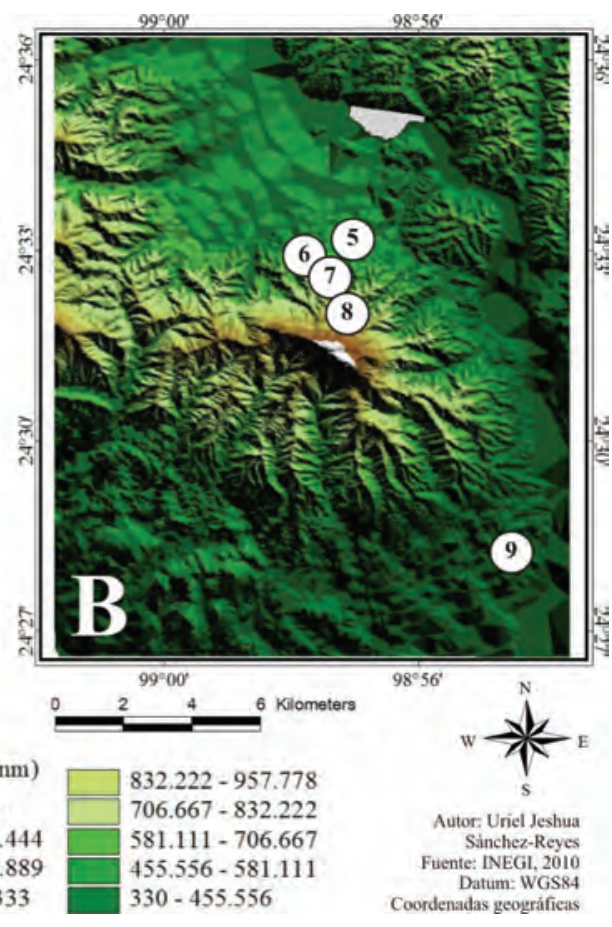

Figura 7. Localización de los nuevos registros de Chrysomelidae en el Cerro El Diente. A) $1=$ Miraces aeneipennis, 2 = Margaridisa atriventris, 3 = Babia costalisdebaja, 4 = Diplacaspis prosternalis. B) $5=$ Acalymma flavovittatum, $6=$ Disonycha teapensis, $7=$ Chrysogramma septempunctata, 8 = Metrioidea varicornis, 9 = Malacorhinus acaciae.

gieren que el número de especies en el área de estudio es superior, ya que se obtuvo una representatividad por debajo del 70\%. Sin embargo, la riqueza de Chrysomelidae en Tamaulipas es mayor a lo señalado por OrdóñezReséndiz et al. (2014), ya que Niño-Maldonado et al. (2005) reportan 138 especies solamente para la Reserva de la Biosfera El Cielo, al sur del estado; por lo tanto, la contribución real del Cerro El Diente a la crisomelofauna de la región no se conocerá hasta que se realice un estudio amplio sobre este grupo en Tamaulipas. Por otra parte, la mayor riqueza de Galerucinae registrada en esta investigación se atribuye a que dicha subfamilia es la que presenta mayor número de especies en México (Ordóñez-Reséndiz et al. 2014) y a nivel mundial (Riley et al. 2002). Además, este patrón se ha observado en los estudios realizados en diversas regiones del país (Andrews \& Gilbert 2005, Niño-Maldonado et al. 2005).

En relación a la diversidad, Margalef (1972) señala que el índice de Shannon toma valores normalmente entre 1.5 y 3.5, excediendo raramente un valor de 4 en comunidades muy diversas. En base a dicha escala, puede establecerse que la diversidad en el Cerro del Diente es elevada $\left(\mathrm{H}^{\prime}=3.78\right)$; esto se atribuye a la baja abundancia registrada, ya que a menor número de individuos se reduce la dominancia y consecuentemente se incrementa la diversidad (Magurran 2004). En otros estudios de Chrysomelidae realizados también en Tamaulipas, Sánchez-Reyes et al. (2014) obtuvieron un valor de 3.54 para el índice de Shannon y 14.6 para el índice de diversidad de Simpson en el Cañón de la Peregrina, mientras que Niño-Maldonado (2000) encontró valores inferiores para ambos índices en varias localidades dentro de la Reserva de la Biósfera el Cielo; a pesar de que los valores de riqueza en dichos estudios fueron superiores a los encontrados en el Cerro el Diente (157 especies en el Cañón de la Peregrina y 145 en la Reserva de la Biosfera el Cielo), la diversidad fue mayor en este trabajo. Esto puede atribuirse a la calidad ecológica del Cerro el Diente, considerada como un área de máxima prioridad para la conservación por sus características bióticas y de alta integridad ecológica (Arriaga et al. 2000, CONABIO et al. 2007). Sin embargo, no se descarta que los valores de diversidad puedan modificarse al incrementarse el periodo de estudio o mediante la realización de un muestreo sistemático que permita emplear los datos de abundancia de las especies. Además, lo anterior permitirá realizar estimaciones de riqueza específica más precisas, ya que el elevado porcentaje de singletons y doubletons se atribuye a la falta de un muestreo más completo (Coddington et al. 2009) e influye en los valores obtenidos de riqueza estimada (Magurran 2004, JiménezValverde \& Hortal 2003).

Variación de Chrysomelidae en el Cerro el Diente. La altitud es una de las variables más relacionadas con la distribución de las especies, generando su disminución con 
el incremento en elevación, o bien, generando la mayor riqueza específica en los estratos altitudinales intermedios (Hodkinson 2005). Sin embargo, en este estudio no se observó un patrón en específico con la altitud, aunque es posible reconocer un incremento de la riqueza a partir de los 550 hasta los $800 \mathrm{msnm}$, ya que en ese intervalo altitudinal fue donde se ubicó el mayor número de especies, en comparación a la primera parte del gradiente (380 a $540 \mathrm{~m}$ ); posteriormente, de los 900 a los $1100 \mathrm{msnm}$ la riqueza disminuye notablemente. El mayor número de especies asociado a altitudes intermedias, tal como se presentó en este trabajo, es un patrón reconocido en insectos en general (Janzen 1973) y se ha observado en otros estudios con Chrysomelidae en Tamaulipas (Sánchez-Reyes et al. 2014) y en otras familias de Coleoptera (Escobar et al. 2005, Fernández et al. 2010).

Entre los factores que se modifican con la altitud y que representan una mayor importancia para las especies de Chrysomelidae, se encuentran el tipo de vegetación y la presencia de las plantas hospederas (Ribeiro et al. 1994, Řehounek 2002, Aslan \& Ayvaz 2009, Flinte et al. 2011). La elevada riqueza específica encontrada en el bosque de encino se puede atribuir al ecotono que se forma en su asociación con el matorral submontano, ya que este último tipo de vegetación se ubica en las laderas de los transectos realizados en el Cerro El diente, y en muchos de los puntos de colecta forma una comunidad vegetal en transición con Quercus sp. y otras especies afines al bosque de encino. Esto podría incidir en la elevada riqueza presentada en ambos tipos de vegetación. Sin embargo, a pesar de que ambas comunidades vegetales registraron el mayor número de especies, fueron también zonas en las que se presentó una baja proporción de especies exclusivas (23\% en el bosque de encino y $0 \%$ en el matorral submontano), indicando que la fauna de ambas comunidades se comparte en mayor medida con el resto de las vegetaciones; esto se corroboró con el índice de similitud, ya que se encontró la mayor semejanza faunística entre el bosque de encino y el matorral submontano, con el mayor número de especies en común. Por otra parte, Pachybrachis sp.3 y Centralaphthona diversa fueron registradas en cuatro tipos de vegetación, lo cual sugiere que ambas especies presentan una mayor tolerancia a las diferentes condiciones ambientales que se presentan en diferentes tipos de vegetación; en forma similar, Sánchez-Reyes et al. (2013) encontraron que $C$. diversa fue la especie más abundante en el Cañón de Peregrina en el municipio de Victoria, Tamaulipas.

Estacionalmente, en el área de estudio se presentan condiciones extremas de temperatura, y los periodos anuales de lluvias se encuentran bien delimitados. En el Cerro del diente, los meses de julio a diciembre constituyen la estación húmeda, lo cual coincide con la mayor riqueza específica observada durante los meses de agosto y septiembre. Esto se ha presentado también en otros estudios, donde las temporadas húmedas y lluviosas favorecen la abundancia y riqueza de especies (Sánchez-Ramos et al. 1993, Jiménez-Sánchez et al. 2011). Sin embargo, en Chrysomelidae y otros escarabajos se ha observado que el mayor número de ejemplares y especies se presenta en las estaciones secas (Sánchez-Reyes et al. 2014); en este caso, es posible que la falta de un muestreo completo anual haya ocultado el patrón estacional real de este grupo.

\section{Nuevos registros}

Holt (2003) señala que la extensión en el rango de distribución de las especies se debe a cambios en factores ecológicos (climáticos, abundancias de especies que interactúan) o a la evolución de las características que influencian los límites del rango (dispersión, características de nicho). Además, para muchos organismos, el rango geográfico es amplio, y la especie se comprende de numerosas poblaciones, aisladas e imposibilitadas a dispersarse debido a la distancia y barreras geográficas (Brown et al. 1996). Sin embargo, dichas conclusiones no pueden aplicarse en este estudio hasta que se realicen inventarios más extensivos y en diferentes áreas geográficas del país, en donde además se analicen las características ambientales que influyen en la distribución de las especies de Chrysomelidae. Es por ello que la ampliación en el rango de distribución de las especies en el Cerro El Diente se atribuye a la carencia de estudios en gran parte del territorio Nacional, que permitan revelar los patrones biogeográficos reales de este grupo en México. En este caso, el aislamiento geográfico y las características de la vegetación en el Cerro El Diente (Martínez 1998, Briones-Villarreal 1991, Arriaga et al. 2000), pueden ser factores importantes que en conjunto generen condiciones de hábitat únicas, permitiendo la existencia de especies que no se habían registrado en estudios previos realizados en Tamaulipas (Niño-Maldonado et al. 2005, Sánchez-Reyes et al. 2014) y otras regiones de México (Andrews \& Gilbert 2005, Furth 2006, 2009, Ordoñez-Reséndiz \& López-Pérez 2009, Ordóñez-Reséndiz et al. 2011, Medvedev et al. 2012). Además, de las 74 especies encontradas, 26 constituyen morfoespecies; dichos ejemplares están en proceso de revisión, por lo que no se descarta la ampliación en la distribución de otras especies.

Finalmente, es necesario señalar que el análisis de abundancia de las especies es de gran importancia en todos los inventarios de biodiversidad y estudios de ecología de comunidades (Hill \& Hamer 1998, Balmer 2002, Chao et al. 2006, McGill et al. 2007). Sin embargo, la omisión 
de dicha información se debe a que el presente estudio constituye un inventario preliminar de la fauna de Chrysomelidae en el Cerro el Diente, en donde la ausencia de un muestreo sistemático aunado al tiempo invertido para los recorridos en cada transecto impidieron la obtención de datos confiables sobre las abundancias de las especies; de la misma forma, la ausencia de otros datos biológicos y ecológicos (información de plantas hospederas, variables ambientales asociadas a las especies) dificulta una conclusión más robusta sobre la influencia de la variación altitudinal y estacional en las especies de este grupo. En cambio, la evaluación realizada constituye una primera etapa en el estudio de la crisomelofauna en el área de estudio, generando un marco de referencia general sobre la riqueza de especies y su presencia en diferentes estratos altitudinales, tipos de vegetación o periodos temporales. Por lo tanto, se espera que los datos aquí presentados sirvan como base para futuras investigaciones en el Cerro el Diente, y además permitan una posterior comparación con otras regiones de Tamaulipas y de México.

Agradecimientos. Se agradece a Ma. del Refugio de la Serna González, Jhanelle Varela de la Serna, Marina Meléndez Vela y Juliana Echartea Echavarría, por su calidad humana y hospitalidad a lo largo de esta investigación en el municipio de San Carlos. A los señores Jesús Gutiérrez y Lauro Meléndez de la Serna por las facilidades brindadas para el acceso al área de estudio. A los revisores anónimos, cuyos acertados comentarios y observaciones permitieron enriquecer en gran medida este manuscrito.

\section{LITERATURA CITADA}

Andrews, F. G. \& Gilbert, A. J. 2005. A preliminary annotated checklist and evaluation of the diversity of the Chrysomelidae (Coleoptera) of the Baja California peninsula, Mexico. Insecta Mundi, 19: 89-116.

Arriaga, L., Espinoza, J. M., Aguilar, C., Martínez, E., Gómez, L. \& Loa, E. 2000. Regiones terrestres prioritarias de México. Comisión Nacional para el Conocimiento y uso de la Biodiversidad, México.

http://www.conabio.gob.mx/conocimiento/regionalizacion/doctos/ Tlistado.html. Fecha de consulta: 20 de abril de 2013.

Aslan, E. G. \& Ayvaz, Y. 2009. Diversity of Alticinae (Coleoptera, Chrysomelidae) in Kasnak Oak Forest Nature Reserve, Isparta, Turkey. Turkish Journal of Zoology, 33: 251-262.

Balmer, O. 2002. Species lists in ecology and conservation: abundance matters. Conservation Biology, 16: 1160-1161.

Baselga, A. \& Novoa, F. 2007. Diversity of Chrysomelidae (Coleoptera) at a mountain range in the limit of the Eurosiberian region, northwest Spain: species richness and beta diversity. Entomologica Fennica, 18: 65-73.

Basset, Y. \& Samuelson, G. A. 1996. Ecological characteristics of an arboreal community of Chrysomelidae in Papua New Guinea. Pp. 243-262. In: P.H.A. Jolivet \& M.L. Cox (Eds.). Chrysomelidae Biology. Volume 2: Ecological Studies. SPB Academic Publishing, Amsterdam.
Blake, D. H. 1955. Revision of the vittate species of the Chrysomelid beetle genus Disonycha from the Americas South of the United States. Proceedings of the United States National Museum, 104: 1-86.

Briones-Villarreal, O. L. 1991. Sobre la flora, vegetación y fitogeografía de la Sierra de San Carlos, Tamaulipas. Acta Botánica Mexicana, 16: 15-43.

Brown, J. H., Stevens, G. C. \& Kaufman, D. M. 1996. The geographic range: size, shape, boundaries, and internal structure. Annual Review of Ecology, Evolution, and Systematics, 27: 597-623.

Burgos-Solorio, A. \& Anaya-Rosales, S. 2004. Los Crisomelinos (Coleoptera: Chrysomelidae: Chrysomelinae) del Estado de Morelos. Acta Zoológica Mexicana (n.s.), 20: 39-66.

Cavazos-Camacho, C. 2000. Evaluación del Bosque Mesófilo de Montaña de San Carlos, Tamaulipas. Universidad Autónoma de Nuevo León, Facultad de Ciencias Forestales. Tesis de Maestría.

Chao, A., Chazdon, R. L., Colwell, R. K. \& Shen, T. J. 2006. Abundance-based similarity indices and their estimation when there are unseen species in samples. Biometrics, 62: 361-371.

Coddington, J. A., Agnarsson, I., Miller, J. A., Kuntner, M. \& Hormiga, G. 2009. Undersampling bias: the null hypothesis for singleton species in tropical arthropod surveys. Journal of Animal Ecology, 78: 573-584.

Colwell, R. K. 2009. EstimateS: Statistical estimation of species richness and shared species from samples. Version 8.2. User's Guide and application. http://purl.oclc.org/estimate. Fecha de consulta: 15 de marzo de 2013.

Comisión Nacional para el Conocimiento y Uso de la Biodiversidad (CONABIO), Comisión Nacional de Áreas Naturales Protegidas (CONANP), The Nature Conservancy - Programa México (TNC) \& Pronatura. 2007. Sitios prioritarios terrestres para la conservación de la biodiversidad, Escala 1: 1000000. México. http://www.conabio.gob.mx/informacion/metadata/gis/spt1mgw. xml?_httpcache $=$ yes\&_xsl $=/ \mathrm{db} /$ metadata $/$ xsl/fgdc_html.xsl\&_ indent $=$ no. Fecha de consulta: 20 de abril de 2013.

Ding, J., Wang, Y. \& Jin, X. 2007. Monitoring populations of Galerucella birmanica (Coleoptera: Chrysomelidae) on Brasenia schreberi and Trapa natans (Lythraceae): implications for biological control. Biological Control, 43: 71-77.

Eben, A. \& Barbercheck, M. E. 1996. Field observation on host plant associations enemies of Diabroticite beetles (Chrysomelidae: Luperini) in Veracruz, Mexico. Acta Zoológica Mexicana (n.s.), 67: 47-65.

Escobar, F., Lobo, J. M. \& Halffter, G. 2005. Altitudinal variation of dung beetle (Scarabaeidae: Scarabaeinae) assemblages in the Colombian Andes. Global Ecology and Biogeography, 14: 327337.

Farrell, B. D. \& Erwin, T. L. 1988. Leaf-beetle community structure in an amazonian rainforest canopy. Pp. 73-90. In: P. Jolivet, E. Petitpierre \& T.H. Hsiao (Eds.). Biology of Chrysomelidae. Kluwer Academic Publishers, Dordrecht, The Netherlands.

Fernández, V., Gamarra, P., Outerelo, R., Cifrián, B. \& Baz, A. 2010. Distribución de estafilíninos necrófilos (Coleoptera, Staphylinidae, Staphylininae) a lo largo de un gradiente altitudinal en la Sierra de Guadarrama, España. Boletín de la Real Sociedad Española de Historia Natural (Sección Biológica), 104: 6186.

Flinte, V., de-Freitas, S., de-Macedo, M. V. \& Monteiro, R.F. 2011. Altitudinal and temporal distribution of Plagiometriona Spaeth, 1899 (Coleoptera, Chrysomelidae, Cassidinae) in a tropical forest in southeast Brazil. ZooKeys, 157: 15-31. 
Flowers, R. W. \& Hanson, P. E. 2003. Leaf beetle (Coleoptera: Chrysomelidae) diversity in eight Costa Rican habitats. Pp. 25-51. In: D.G. Furth (Ed). Special topics in leaf beetle biology, Proceedings of the 5th International Symposium on the Chrysomelidae. Pensoft Publishers, Sofia-Moscow.

Furth, D. G. 2004. Alticinae (Coleoptera: Chrysomelidae). Pp. 669684. In: J.E. Llorente-Bousquets, J.J. Morrone, O. Yáñez-Ordóñez \& I. Vargas-Fernández (Eds). Biodiversidad, taxonomía y biogeografía de artrópodos de México: hacia una síntesis de su conocimiento Vol. IV. UNAM-CONABIO, México.

Furth, D. G. 2006. The Current Status of Knowledge of the Alticinae of Mexico (Coleoptera: Chrysomelidae). Bonner zoologische Beiträge, 54: 209-237.

Furth, D. G. 2009. Flea beetle diversity of the Sierra Tarahumara, Copper Canyon, Mexico (Chrysomelidae: Alticinae). Pp. 131-151. In: P. Jolivet, M. Schmitt \& J. Santiago-Blay (Eds.). Research on Chrysomelidae Volume 2. Koninklijke Brill, Leiden, The Netherlands.

Furth, D. G. 2013. Diversity of Alticinae in Oaxaca, Mexico: A preliminary study (Coleoptera, Chrysomelidae). ZooKeys, 332: 1-32.

Furth, D. G. \& Savini, V. 1996. Checklist of the Alticinae of Central America, including Mexico (Coleoptera: Chrysomelidae). Insecta Mundi, 10: 45-68.

Furth, D. G., Longino, J. T. \& Paniagua, M. 2003. Survey and Quantitative Assessment of Flea Beetle Diversity in a Costa Rican Rainforest (Coleoptera: Chrysomelidae: Alticinae). Pp. 1-23. In: D.G. Furth (Ed). Special Topics in Leaf Beetle Biology, Proceedings of the 5th International Symposium on the Chrysomelidae. Pensoft Publishers, Bulgaria.

Gotelli, N. J. \& Colwell, R. K. 2011. Chapter 4. Estimating species richness. Pp. 39-54. In: A.E. Magurran \& B.J. McGill (Eds.). Biological Diversity: frontiers in measurement and assessment. Oxford University Press, New York.

Hammer, Ø., Harper, D. A. T. \& Ryan, P. D. 2001. PAST: Paleontological Statistics Software Package for Education and Data Analysis. Palaeontologia Electronica, 4: 1-9.

Hill, J. K. \& Hamer, K. C. 1998. Using species abundance models as indicators of habitat disturbance in tropical forests. Journal of Applied Ecology, 35: 458-460.

Hodkinson, I. D. 2005. Terrestrial insects along elevation gradients: species and community responses to altitude. Biological Reviews of the Cambridge Philosophical Society, 80: 489-513.

Holt, R. D. 2003. On the evolutionary ecology of species' ranges. Evolutionary Ecology Research, 5: 159-178.

Hortal, J., Borges, P. A. V. \& Gaspar, C. 2006. Evaluating the performance of species richness estimators: sensitivity to sample grain size. Journal of Animal Ecology, 75: 274-287.

Janzen, D. H. 1973. Sweep samples of tropical foliage insects: effects of seasons, vegetation types, elevation, time of day, and insularity. Ecology, 54: 687-708.

Jiménez-Sánchez, E., Juárez-Gaytán, O. M. \& Padilla-Ramírez, J. R. 2011. Estafilínidos (Coleoptera: Staphylinidae) necrófilos de Malinalco, Estado de México. Dugesiana, 18: 73-84.

Jiménez-Valverde, A. \& Hortal, J. 2003. Las curvas de acumulación de especies y la necesidad de evaluar la calidad de los inventarios biológicos. Revista Ibérica de Aracnología, 8: 151-161.

Jolivet, P., Santiago-Blay, J. A. \& Schmitt, M. 2009. Research on Chrysomelidae, Volume 2. Brill, Leiden, The Netherlands.

Jost, L. 2006. Entropy and diversity. Oikos, 113: 363-375.

Kalaichelvan, T. \& Verma, K. K. 2005. Checklist of Leaf Beetles (Coleoptera: Chrysomelidae) of Bhilai-Durg. Zoos' Print Journal, 20: $1838-1842$.
Linzmeier, A. M., Ribeiro-Costa, C. S. \& Marinoni, R. C. 2006. Fauna de Alticini (Newman) (Coleoptera, Chrysomelidae, Galerucinae) em diferentes estágios sucessionais na Floresta com Araucária do Paraná, Brasil: diversidade e estimativa de riqueza de espécies. Revista Brasileira de Entomologia, 50: 101-109.

Magurran, A. E. 2004. Measuring Biological Diversity. Blackwell Science Ltd, Oxford, UK.

Margalef, R. 1972. Homage to Evelyn Hutchinson, or why is there an upper limit to diversity? Transactions of the Connecticut Academy of Arts and Sciences, 44: 211-235.

Martínez, M. 1998. Inventario florístico de la Sierra de San Carlos, Tamps. Universidad Autónoma de Tamaulipas. Instituto de Ecología Aplicada. Informe final SNIB-CONABIO proyecto No. P024. México, D.F.

Martínez-Sánchez, I., Niño-Maldonado, S., Carreón-Pérez, A. \& Horta-Vega, J. V. 2010. Nuevos registros de Cassidinae (Coleoptera: Chrysomelidae) para el Estado de Hidalgo, México. Entomología Mexicana, 9: 921-924.

McGill, B. J., Etienne, R. S., Gray, J. S., Alonso, D., Anderson, M. J., Benecha, H. K., Dornelas, M., Enquist, B. J., Green, J. L., He, F., Hurlbert, A. H., Magurran, A. E., Marquet, P. A., Maurer, B. A., Ostling, A., Soykan, C. U., Ugland, K. I. \& White, E. P. 2007. Species abundance distributions: moving beyond single prediction theories to integration within an ecological framework. Ecology Letters, 10: 995-1015.

Medvedev, L. N., Niño-Maldonado, S., Sánchez-Reyes, U. J. \& Moseyko, A. G. 2012. To the knowledge of Mexican Clytrini (Chrysomelidae, Cryptocephalinae), with description of two new species of the genus Coscinoptera Lacordaire, 1848. Zoosystematica Rossica, 21: 244-253.

Meissle, M., Pilz, C. \& Romeis, J. 2009. Susceptibility of Diabrotica virgifera virgifera (Coleoptera: Chrysomelidae) to the entomopathogenic fungus Metarhizium anisopliae when feding on Bacillus thuringiensis Cry3Bb1-Expressing Maize. Applied and Environmental Microbiology, 75: 3937-3943.

Moldenke, R. A. 1970. A revision of the Clytrinae of North America North of the Isthmus of Panama (Coleoptera: Chrysomelidae). Stanford University, California.

Munroe, D. D. \& Smith, R. F. 1980. A revision of the systematics of Acalymma sensu stricto Barber (Coleoptera: Chrysomelidae) from North America including Mexico. Memoirs of the Entomological Society of Canada, 112: 1-92.

Niño-Maldonado, S. 2000. Diversidad de Chrysomelidae (Coleoptera) en el Bosque Mesófilo de la Reserva "El Cielo", Gómez Farías, Tamaulipas. Tesis de Doctorado. Universidad Autónoma de Tamaulipas. Unidad Académica, Multidisciplinaria Agronomía y Ciencias. Cd. Victoria, Tamaulipas. México.

Niño-Maldonado, S., Riley, E. G., Furth, D. G. \& Jones, R. W. 2005. Coleoptera: Chrysomelidae. Pp. 417-425. In: G. SánchezRamos, P. Reyes-Castillo \& R. Dirzo (Eds.). Historia Natural de la Reserva de la Biósfera El Cielo, Tamaulipas, México. Universidad Autónoma de Tamaulipas, México.

Ordóñez-Reséndiz, M. M. \& López-Pérez, S. 2009. Crisomélidos (Coleoptera: Chrysomelidae) de las Sierras de Taxco-Huautla, México. Entomología Mexicana, 8: 946-951.

Ordóñez-Reséndiz, M. M., López-Pérez, S. \& Rodríguez-Mirón, G. 2011. Chrysomelidae (Coleoptera) en la Sierra Nevada, México. Entomología Mexicana, 10: 779-784.

Ordóñez-Reséndiz, M. M., López-Pérez, S. \& Rodríguez-Mirón, G. 2014. Biodiversidad de Chrysomelidae (Coleoptera) en México. Revista Mexicana de Biodiversidad, Supl., 85: 271-278. 
Řehounek, J. 2002. Comparative study of the leaf beetles (Coleoptera: Chrysomelidae) in chosen localities in the district of Nymburk. Acta Universitatis Palackianae Olomucensis Facultas Rerum Naturalium Biologica, 39-40: 123-130.

Ribeiro, S. P., Carneiro, M. A. A. \& Fernandes, O. W. 1994. Distribution of Brachypnoea (Coleoptera: Chrysomelidae) in an altitudinal gradient in a Brazilian savanna vegetation. Phytophaga, 6: 29-33.

Riley, E. G., Clark, S. M. \& Seeno, T. N. 2003. Catalog of the leaf beetles of America north of Mexico. Special publication no. 1. Coleopterists Society.

Riley, E. G., Clark, S. M., Flowers, R. W. \& Gilbert, A. J. 2002. 124. Chrysomelidae Latreille 1802. Pp. 617-691. In: R.H. Arnett Jr., M.C. Thomas, P.E. Skelley \& J.H. Frank (Eds.). American Beetles Volume II. Polyphaga: Scarabaeoidea through Curculionoidea. CRC. Press LLC, Boca Raton, Florida.

Sánchez-Ramos, G., Lobo, J., Lara-Villalón, M. \& Reyes-Castillo, P. 1993. Distribución altitudinal y estacional de la entomofauna necrófila en la Reserva de la Biósfera "El Cielo", Tamaulipas, México. BIOTAM, 5: 13-24.

Sánchez-Reyes, U. J., Niño-Maldonado, S., Barrientos-Lozano, L., Banda-Hernández, J. E. \& Medina-Puente, A. 2013. Galerucinae (Coleoptera: Chrysomelidae) del Cañón de la Peregrina,
Victoria, Tamaulipas, México. Entomología Mexicana, 12: 15171522.

Sánchez-Reyes, U. J., Niño-Maldonado, S. \& Jones, R. W. 2014. Diversity and altitudinal distribution of Chrysomelidae (Coleoptera) in Peregrina Canyon, Tamaulipas, Mexico. ZooKeys, 417: 103-132.

Staines, C. L. \& Staines, S. L. 2001. The leaf beetles (Insecta: Coleoptera: Chrysomelidae): Potential indicator species assemblages for natural area monitoring. Pp. 233-244. In: G.D. Therres (Ed.). Proceedings of Conservation of biological diversity. A key to restoration of the Chesapeake Bay ecosystem and beyond. Maryland Department of Natural Resources.

Syrett, P., Fowler, S. V. \& Emberson, R. M. 1996. Are chrysomelid beetles effective agents for biological control of weeds? Pp. 399407. In: V.C. Moran \& J.H. Hoffmann (Eds.). Proceedings of the IX International Symposium on Biological Control. University of Cape Town, Stellenbosch, South Africa.

Wilcox, J. A. 1951. A New Species and New Genus of Galerucinae (Chrysomelidae: Coleoptera). The Ohio Journal of Science, 51: 90-94.

Wilcox, J. A. 1975. Checklist of the beetles of Canada, United States, Mexico, Central America and the West Indies. Vol. 1, pt.7. The leaf beetles (red version). Biological Research Institute of America, New York. 